Due to the widespread use of sensors and sensor networks in the tasks of territory coverage, the relevant criteria are maximizing coverage and minimizing energy consumption. At the same time, the compliance of the network with these criteria is an urgent problem in the modern technological world. A modification of the method for constructing energy-efficient sensor networks is proposed by introducing an additional criterion for minimizing the number of sensors and limiting the number of sensors used, which allows reducing the energy consumption of sensor networks by $19 \%$. In the resulting optimization problem, the optimality criteria are the functions of minimizing the area of uncovered territory, the value of energy consumption, and the number of sensors. The optimum solution is formed by pairs of values of the coverage radius and the level of intersection of the coverage areas, which provide maximum coverage while minimizing energy consumption and the number of sensors used. To solve the problem, the parameter convolution method and the genetic algorithm were used. In the case of dynamic sensors, the problem is to find such a trajectory of the sensor that provides the maximum flyby of the territory with a minimum length. A grid algorithm is proposed to find the necessary trajectory. The presented algorithm consists in dividing the territory into nodes and estimating the value of the covered territory by the sensor in this node. After the formation of estimates, the search for a Hamiltonian path was used. The case of a multiply connected territo$r y$ with the possibility of turning it into a simply connected one is considered. A scheme for finding the parameters of energy-efficient coverage of the territory using static and dynamic sensors is proposed

Keywords: sensor network, territory coverage, energy efficiency of sensor networks, optimum flight trajectory

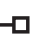

口-
UDC 004.94

DOI: $10.15587 / 1729-4061.2022 .252988$

\title{
DEVELOPMENT OF A MODIFICATION OF THE METHOD FOR CONSTRUCTING ENERGY-EFFICIENT SENSOR NETWORKS USING STATIC AND DYNAMIC SENSORS
}

\author{
Volodym yr Petrivskyi \\ Postgraduate Student* \\ Viktor Shevchenko \\ Doctor of Technical Sciences, Professor* \\ Serhi Y $\mathbf{Y}$ s e iev \\ Corresponding author
}

Doctor of Technical Sciences, Professor***

E-mail: Serhii_Yevseiev@khpi.edu.ua

Oleksandr Milov

Doctor of Technical Sciences, Professor***

O leks andr Laptiev

Doctor of Technical Sciences, Associate Professor, Senior Researcher Department of Cyber Security and Information Protection**

Oleksi i Bychov

Doctor of Technical Sciences, Professor*

Vitalii Fedoriienko

Senior Researcher

Section of Information Technology Development Department of Information Technology Development and Implementation of Informatization Projects of the Armed Forces of Ukraine of the Center for Military Strategic Studies The National Defence University of Ukraine named after Ivan Cherniakhovskyi Povitroflotskyi ave., 28, Kyiv, Ukraine, 03049

Maksim Tkachenko $\mathrm{PhD}$ *

O leg Kurchenko PhD, Associate Professor, Senior Researcher* Ivan Opirsky

Doctor of Technical Sciences, Professor

Department of Information Security

Lviv Polytechnic National University

S. Bandery, str., 12, Lviv, Ukraine, 79013

*Department of Programming and Computer Equipment** **Taras Shevchenko National University of Kyiv

Volodymyrska str., 60, Kyiv, Ukraine, 01033

***Department of Cyber Security

National Technical University «Kharkiv Polytechnic Institute»

Kyrpychva str., 2, Kharkiv, Ukraine, 61002

Received date 10.01.2022 Accepted date 16.02.2022 Published date 28.02.2022
How to Cite: Petrivskyi, V., Shevchenko, V., Yevseiev, S., Milov, O., Laptiev, O., Bychkov, O., Fedoriienko, V., Tkachenko, M., Kurchenko, O., Opirskyy, I. (2022). Development of a modification of the method for constructing energy-efficient sensor networks using static and dynamic sensors. Eastern-European Journal of Enterprise Technologies, 1 (9 (115)), 15-23. doi: https://doi.org/10.15587/1729-4061.2022.252988
1. Introduction

Sensors and sensor networks are widespread in all spheres of human activity. The use of sensors is provided by their ad- vantages, such as small size, various measured values, ease of software development, low power consumption, mobility, service life. Also, many tasks, such as collecting, analyzing, identifying and measuring data, can be solved using sensors [1, 2]. 
In addition, sensors are an integral part of the Internet of Things and the Internet of Everything technolo gies [3, 4].

In practice, the key criteria for building sensor networks in the monitoring task are territory coverage, energy efficiency and the total cost of the network. The maximum coverage of the territory is achieved by placing additional sensors and increasing the coverage radius of the sensors. An increase in the coverage radius increases power consumption, and an increase in the number of sensors increases the total cost of the network and its maintenance. With dynamic sensors, the maximum coverage is achieved by flying over the entire territory. Also, in this case, the sensor spends battery power not only for collecting information, but also for movement. Obviously, minimizing the length of the flight path will reduce the power consumption of the sensor and significantly increase the battery life. Also noteworthy is the reduction in the sensor size, resulting in a decrease in the device's battery size. In turn, the price of high-tech sensors increases.

Currently, there are a number of studies on the construction of sensor networks, taking into account the criteria for maximizing the coverage, total cost and energy efficiency of networks. But most of them take into account only one of the criteria and do not take into account the possibility of changing the coverage radius during the sensor movement. The development of a method for finding optimum network parameters, which simultaneously takes into account the criteria for maximizing coverage, minimizing energy consumption and the number of sensors, changing the coverage radius during movement, is an urgent scientific task. The solution of the described problem will make it possible to find the parameters of sensor networks that will provide maximum coverage of the territory with minimal energy consumption, which, in turn, will increase the network life.

\section{Literature review and problem statement}

The paper [5] proposes a method for optimum coverage of the territory with sensors of different ranges. To solve this problem, the authors modified the least squares method. The optimality criterion is the maximum coverage of the territory with a minimum intersection of the coverage areas. But the paper does not take into account the power consumption of sensors, and reducing the intersection of coverage areas can lead to the formation of uncovered areas. Optimizing the location of sensors to solve the problem of critical network coverage with the goals of accuracy and cost is proposed in [6]. The issue of the energy efficiency of the coverage remains untouched. An algorithm for covering a territory of arbitrary geometry with sensors with a coverage radius by approximating the territory with an orthogonal polygon is presented in [7]. The disadvantage is the location of sensors without the possibility of crossing the coverage areas, which will result in the impossibility to transmit information among the sensors and the presence of uncovered areas.

The paper [8] considers the problem of energy-efficient regular coverage of a flat area with sensors with two selected observation radii. The known results on the coverage quality are improved, and the total energy consumption for monitoring and data transmission among the sensor network elements is optimized. But the issue of the possibility of regulating the coverage radii and the intersection area of the sensors remained unresolved. The paper [9] proposes a method for minimizing the energy consumption of the sensor network by regulating the coverage radii. The disadvantages of the proposed method are the use of a fixed value of the intersection level of the coverage areas and a decrease in the total network coverage.

An approach to minimizing the power consumption of mobile sensors by adjusting the coverage radii is described in [10]. The authors do not take into account the maximization of territory coverage during sensor movement. The paper [11] presents algorithms for constructing the sensor flight path of minimum length with a maximum accumulation of information, but the flyby of the entire territory is not taken into account and hard-to-reach areas are missed. The clustering method proposed in [12] is based on the midpoint method, taking into account the residual energy and the distance between nodes. But this approach does not provide maximum territory coverage. The search for the flight path of the sensor as a traveling salesman problem is presented in [13]. The proposed approach does not provide coverage of the entire territory. In [14], for efficient runoff-based data collection, the trajectory of mobile runoff based on the Moore curve was considered. In this case, the sensor coverage radius is not taken into account, which will result in a flyby of already covered areas of the territory. The algorithm for constructing the motion trajectory of the sensor based on pseudo-shape control is presented in [15]. The proposed algorithm does not take into account the sensor coverage radius and the possibility of capturing the area outside the required territory.

Thus, an unresolved problem in the construction of sensor networks is to simultaneously take into account the criteria for maximizing coverage, minimizing the power consumption of the sensor network, reducing the number of sensors, and also finding the optimum sensor flight path, taking into account the coverage of the territory. The proposed approach to modifying the method of building energy-efficient sensor networks allows maximizing the coverage area, minimizing the network power consumption, and reducing the number of sensors used.

\section{The aim and objectives of the study}

The aim of the study is to develop a modification of the method for constructing energy-efficient sensor networks using static and dynamic sensors. This approach allows minimizing the number of sensors involved when using static sensors, as well as finding the optimum trajectory for flying around the territory when using dynamic sensors. This will make it possible to build sensor networks and flight paths of the territory that meet the criteria for maximizing coverage and minimizing power consumption.

To achieve the aim, the following objectives were set:

- to develop a modified method for building a sensor network in conditions of maximizing coverage while minimizing power consumption;

- to develop a method for constructing the trajectory of motion of dynamic sensors, taking into account the minimization of power consumption;

- to develop an algorithm for finding the parameters of an energy-efficient sensor network using static and dynamic sensors.

\section{Research materials and methods}

To solve the problem of developing a method for constructing a sensor network in conditions of maximizing coverage 
while minimizing power consumption, taking into account the limitation in the number of sensors, the method presented in [16] was taken as a basis. The method consists in solving a non-linear multicriteria optimization problem of finding the coverage radius and intersection areas of sensors, which provide the maximum territory coverage with minimum power consumption. To solve the formulated problem, the method of linear convolution of criteria was used, which involves converting a set of available partial criteria into one supercriterion by summing criteria with weight factors, the sum of which is equal to one [18]. A genetic algorithm was also used, which is aimed to solve optimization and modeling problems by random selection, combination and variation of the desired parameters using mechanisms similar to natural selection in nature [19].

When searching for the optimum path in the problem of developing a method for constructing the motion trajectory of sensors, taking into account the minimization of energy consumption, the search for a Hamiltonian path was used. In graph theory, a Hamiltonian path is a loop-free path passing through each vertex of the graph exactly once [20]. In the case where the start and end points coincide, we will look for a Hamiltonian cycle. To find the Hamiltonian path, the branch and bound method was used.

\section{Methods and algorithms for constructing energy- efficient sensor networks using static and dynamic sensors}

5. 1. Development of a modified method for constructing a sensor network in conditions of maximizing coverage while minimizing power consumption

Some territory is considered that needs to be covered with sensors, that is, to build a sensor network. Among all the sensor characteristics (size, shape, coordinates, coverage radius, battery volume, measurement value), only the coordinates and coverage radius were taken into account. As a result, each sensor can be represented as follows (1):

$$
s_{i}=s_{i}\left(x_{i}, y_{i}, r_{i}\right)
$$

where $x_{i}, y_{i}$ are the sensor coordinates, $r_{i}$ is the coverage radius, $1 \leq i \leq n, n$ is the number of sensors.

To find the territory coverage, we use the method presented in [16], which is formulated as an optimization problem of the following form:

$$
\begin{aligned}
& \left\{\begin{array}{l}
E(r) \rightarrow \min , \\
Z(r, c) \rightarrow \min ,
\end{array}\right. \\
& 0 \leq r \leq r^{\max }, \\
& c_{\min } \leq c \leq r^{\max },
\end{aligned}
$$

where $E(r)$ is power consumption, $Z(r, c)$ is the area of uncovered territory, $r$ is the coverage radius, $c$ is the intersection of the coverage areas.

The energy consumption according to [16] will be:

$$
E(r)=r^{2} .
$$

The function $Z(r, c)$ in equation (2) is presented as the area of uncovered territory by sensors with a coverage radius $r$ and the intersection level of the coverage areas as follows:

$$
Z(r, c)=Z_{\text {cov }}(r)-Z_{\text {int }}(r, c),
$$

where $Z_{\text {cov }}=\pi r^{2}$.

The value of the intersection area was calculated using the approach presented in [17]:

$$
Z_{\text {int }}(r, c)=m \frac{r^{2}}{2}(K(r, c)-\sin (K(r, c))),
$$

where $r$ is the sensor coverage radius, $c$ is the intersection level of the coverage areas, $r^{\max }$ is the maximum coverage radius value, $c_{\min }$ is the minimum intersection value of coverage radii, $K(r, c)=2 \arccos (\alpha / r), \alpha=r-c / 2, m$ is the number of intersections. Schematically, the intersection level of the coverage areas $c$ and the uncovered area $Z(r, c)$ can be represented as follows (Fig. 1).

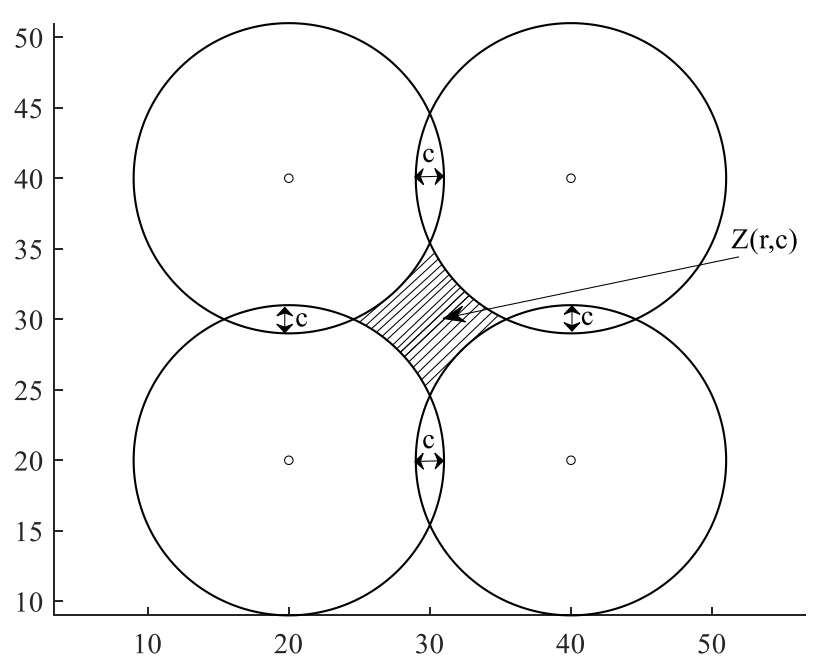

Fig. 1. Intersection level of the coverage areas $c$ and the function $Z(r, c)$

The disadvantage of this method is the possibility of using an unlimited number of sensors. In practice, the number of sensors available for use is fixed, and it is obvious that it is economically advantageous to use a minimum number of sensors. With this in mind, we introduce a function of the number of sensors into system (2), which will be calculated as the integer part of dividing the area of the entire territory by the area covered by one sensor:

$$
N(r)=\left[\frac{T_{s}}{\pi r^{2}}\right],
$$

where $T_{s}$ is the area of the territory to be covered.

We denote as $N_{\max }$ the number of sensors available for use and take this value into account as an additional constraint when solving the problem.

Thus, method (2)-(7) is modified to minimize the number of sensors used and limit the number of available sensors and is formulated as a problem:

$$
\begin{aligned}
& \left\{\begin{array}{l}
E(r) \rightarrow \min , \\
Z(r, c) \rightarrow \min , \\
N(r) \rightarrow \min ,
\end{array}\right. \\
& 0 \leq r \leq r^{\max }, \\
& c_{\min } \leq c \leq r^{\max }
\end{aligned}
$$


or

$$
\begin{aligned}
& c=\text { const, } \\
& 0 \leq N(r) \leq N_{\max } .
\end{aligned}
$$

In equation (9), $E(r)$ is a function of energy consumption (5), $Z(r, c)$ is a function of uncovered territory (6), and $N(r)$ is a function of the number of sensors (8), $N_{\max }$ is the number of sensors available for use. The formulated problem takes into account the criteria for minimizing energy consumption, the area of uncovered territory and the number of sensors used. It also takes into account the limitation of the number of sensors available for use, the possibility of regulating the coverage radii and the intersection level of the coverage areas.

Using the parameter convolution method, the problem of minimizing power consumption with maximum coverage, taking into account the intersection of the sensor coverage areas, is presented in the following form:

$$
\begin{aligned}
& F(r, c)=\alpha_{1} Z(r, c)+\alpha_{2} E(r)+\alpha_{3} N(r), \\
& 0 \leq r \leq r^{\max }, \\
& c_{\min } \leq c \leq r^{\max }
\end{aligned}
$$

or

$$
\begin{aligned}
& c=\text { const, } \\
& 0 \leq N(r) \leq N_{\max } . \\
& \alpha_{1}+\alpha_{2}+\alpha_{3}=1, \\
& 0 \leq \alpha_{1} \leq 1,0 \leq \alpha_{2} \leq 1,0 \leq \alpha_{3} \leq 1,
\end{aligned}
$$

where $r$ is the sensor coverage radius, $c$ is the intersection level of the coverage areas, $r^{\max }$ is the maximum coverage radius value, $c_{\min }$ is the minimum intersection value of coverage radii, $N_{\max }$ is the number of available sensors, $\alpha_{1}, \alpha_{2}, \alpha_{3}$ are expert estimates of parameters significance.

The solution of the stated problem in the form of (9)-(12) or (13)-(18) will be a set of Pareto-optimum combinations of coverage radii and intersection level of coverage radii, which provide the maximum coverage of the territory with minimum energy consumption.

Thus, the algorithm of the method for constructing a sensor network under conditions of maximizing coverage while minimizing power consumption, taking into account the limitation in the number of sensors, can be schematically represented as follows (Fig. 2).

The first step is to enter the territory area, the maximum coverage radius, the intersection level of the coverage areas and the number of sensors available for use. You can also upload a territory image from a file and use automatic capture of territory limits and area calculation. Next, you need to choose a method for solving the problem: a genetic algorithm or a parameter convolution method. The result will be a set of Pareto-optimum combinations of coverage radii and intersection level of coverage radii. Among the results obtained, it is necessary to choose a solution that satisfies the user and build the appropriate coverage of the territory.

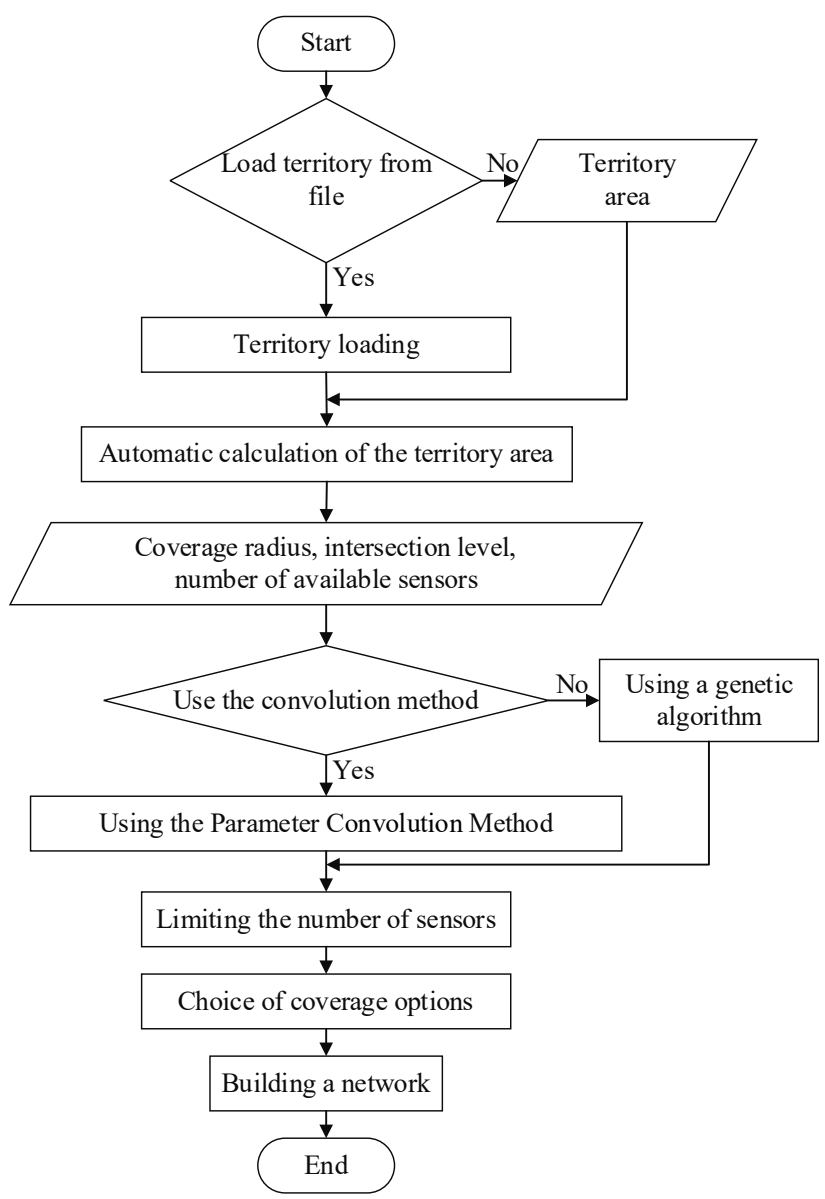

Fig. 2. Block diagram of building a sensor network in conditions of maximizing coverage while minimizing power consumption, taking into account the limitation of the number of sensors

5. 2. Development of a method for constructing the motion trajectory of dynamic sensors, taking into account the minimization of energy consumption

The use of dynamic sensors is considered. In the case of dynamic sensors, the maximum coverage will mean the maximum coverage of the territory during the flyby, and, as a result, the problem of finding the optimum sensor flight path. Since the sensor consumes battery power for movement, the optimality criterion will be minimizing the length of the trajectory. Another optimality criterion will be the maximization of the covered territory. Thus, the problem of constructing the optimum trajectory of the sensor movement was formulated taking into account the minimization of energy consumption in the following form:

$$
\begin{aligned}
& \left\{\begin{array}{l}
L(x) \rightarrow \min , \\
S(x) \rightarrow \max ,
\end{array}\right. \\
& L(x)=\sum_{i=1}^{n} \sum_{j=1}^{n} c_{i j} x_{i j}, \\
& S(x)=\sum_{i=1}^{n} \alpha_{i}\left(\pi R^{2}\left(x_{i}\right)-S_{\text {out }}\left(x_{i}\right)\right), \\
& \sum_{i=1}^{n} x_{i j}=1, j=\overline{1, n} \\
& \sum_{j=1}^{n} x_{i j}=1, i=\overline{1, n},
\end{aligned}
$$




$$
u_{i}-u_{j}+(n-1) x_{i j} \leq n-2, i, j=\overline{2, n}, i \neq j,
$$

$x_{i j}=\left\{\begin{array}{l}1, \text { if we follow from point } i \text { to point } j, \\ 0, \text { if we do not follow from point } i \text { to point } j,\end{array}\right.$

$\alpha_{i}=\left\{\begin{array}{l}1, \text { if point } i \text { belongs tp the trajectory, } \\ 0, \text { if point } i \text { does not belong to the trajectory. }\end{array}\right.$

In the formulation of the problem (19)-(26), $S(x)$ is the sum of the sensor coverage area at each route point $x_{i}$. The elements of the sum are calculated as a difference between the total area covered by the sensor at a given point with a radius $R\left(x_{i}\right)$ and the area covered outside the territory $S_{\text {out }}\left(x_{i}\right)$. The value of $S_{\text {out }}\left(x_{i}\right)$ is calculated using the approach proposed in [17]. Condition (22) means only one exit from the point, and condition (23) means only one entry to the point, that is, each point is visited only once. Constraint (24) is a special condition providing the closure of routes and the absence of unrelated subcycles. The values $c_{i j}$ are the distance between the points through which the trajectory must pass. The distance between the points was calculated as Euclidean distance.

To solve the problem and build an optimum trajectory, we transfer the territory to the $X O Y$ coordinate plane so that the territory is in contact with the $O X$ and $O Y$ axes at one point to each axis. After transferring to the coordinate plane, the plane was covered with a uniform grid with a step equal to the coverage radius $r$. For further use, the territory was designated as $T$.

In the next step, the grid nodes were numbered starting from one along the $O X$ axis and the vector $A$ was formed, the elements of which are the corresponding grid nodes. The value of the vector elements will be equal to the area of the territory $s_{i}$ covered by the sensor at a given point, or zero if the grid node does not belong to the territory $T$ :

$$
a_{i}=\left\{\begin{array}{l}
s_{i}, a_{i} \in T, \\
0, a_{i} \notin T .
\end{array}\right.
$$

The values of $s_{i}$ were calculated using Pick's theorem [21]. To use Pick's theorem, it is necessary to additionally build a grid with a small step in the square described around a given sensor position. The points of this square will have the $\left(x_{c}-R, y_{c}-R\right),\left(x_{c}-R, y_{c}+R\right),\left(x_{c}+R, y_{c}+R\right),\left(x_{c}+R, y_{c}-R\right)$ coordinates, respectively.

Also, the values of the vector $A$ elements will be zero in the case when the value of the covered area at a given grid node is less than a certain expert value of the minimum allowable coverage area, which is designated as $S_{\min }$ :

$$
a_{i}=\left\{\begin{array}{l}
s_{i}, a_{i} \in T, \\
0, a_{i} \notin T \vee s_{i} \leq S_{\text {min }} .
\end{array}\right.
$$

If it is possible to adjust the sensor coverage radius, the minimum and maximum sensor coverage radii are designated as $r_{\min }$ and $r_{\max }$. In the case when, with a continuous coverage radius, the sensor covers an area outside the territory under consideration, a coverage radius was used in which the area covered by the sensor does not go beyond the territory under consideration. For trajectory construction, the maximum allowable radius was used. Adjusting the coverage radius will also reduce the power consumption of the sensor. In this case, in addition to the node vector, the coverage ra- dius vector $R$ is formed, the elements of which will be equal to $r_{\min } \leq r_{i} \leq r_{\max }$.

At the next stage, a matrix of transitions between nodes $P$ was formed. The elements of this matrix $p_{i j}$ will be equal to one in the case when $a_{i}>0$ and $a_{j}>0$. In the case when $a_{i}=0$ or $a_{j}=0$, the value of $p_{i j}$ will be zero:

$$
p_{i j}=\left\{\begin{array}{l}
1, a_{i}>0 \wedge a_{j}>0, \\
0, a_{i}=0 \vee a_{j}=0 .
\end{array}\right.
$$

The algorithm for forming the transition matrix can be represented by the following scheme (Fig. 3).

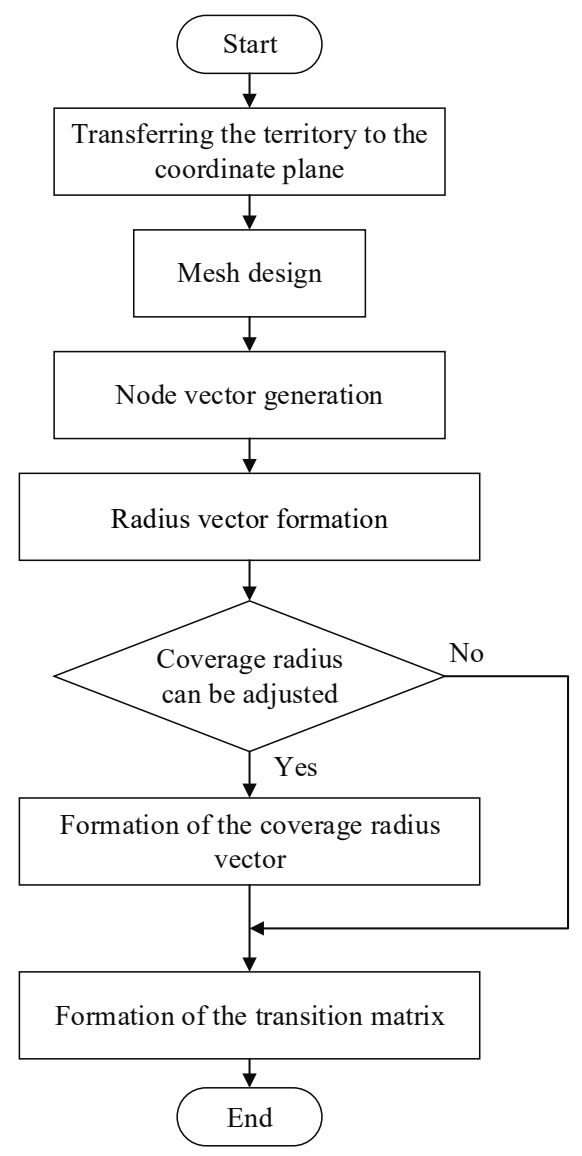

Fig. 3. Block diagram of the transition matrix formation algorithm

After forming the matrix $P$, the task is to find a Hamiltonian path [20], that is, a path passing through each node only once.

The case of a multiply connected domain is considered. In this case, the trajectory constructed using the above algorithm will not cover the entire area considered. To ensure the completeness of the coverage, the domain was reduced to a simply connected one by combining the domains. This combination was carried out using additional transitions between nodes by adding one connection between the nearest nodes for each pair of simply connected domains. Fig. 4, $a$ shows a doubly connected domain. To turn it into a simply connected one (Fig. $4, b$ ), it is necessary to add a transition between nodes 103 and 113, that is, make the values of the corresponding elements of the transition matrix equal to one: $p_{39.45}=1$ and $p_{45.39}=1$. Schematically, this can be shown as follows (Fig. 4). 
In Fig. 4, red dots indicate grid nodes, transitions between nodes are indicated by red arrows. According to the described algorithm, the presented territory will not be simply connected (Fig. 4, $a$ ). To ensure that the domain is simply connected, it is necessary to add a connection between grid nodes 103 and 113 (Fig. 4, $b$ ).
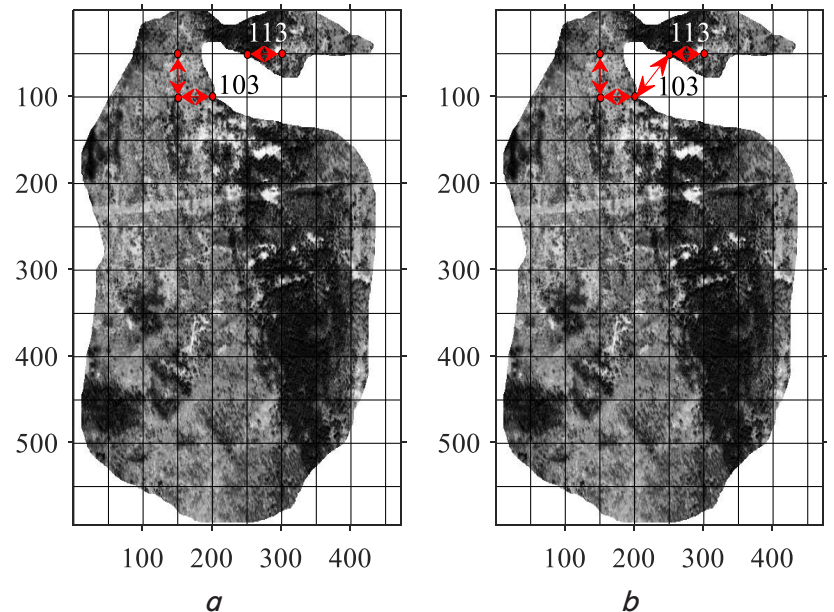

$100 \quad 200 \quad 300 \quad 400$ $b$

Fig. 4. Example of bringing a doubly connected domain to a simply connected one by adding a connection between nodes 103 and 113: $a$ - doubly connected domain; $b-$ simply connected domain

Thus, the grid method algorithm can be schematically represented as follows (Fig. 5).

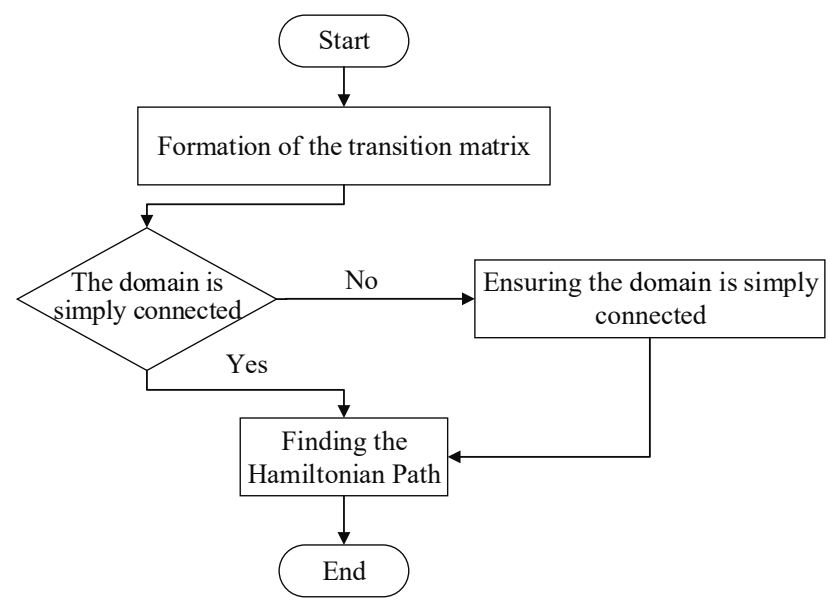

Fig. 5. Block diagram of the grid algorithm

At the first stage of the algorithm, a matrix of transitions between nodes is formed according to the algorithm shown in Fig. 2. After checking and, if necessary, ensuring that the domain is simply connected, a Hamiltonian path from the first node to the last one is sought.

The result of the algorithm will be a vector of nodes in the flyby sequence. If it is necessary to move the sensor along a cyclic trajectory, the start node and the end node must match.

5. 3. Development of an algorithm for finding the parameters of an energy-efficient sensor network using static and dynamic sensors

The practical implementation of the modified method for constructing energy-efficient sensor networks and the method for constructing the trajectory of sensors, taking into account the minimization of energy consumption, allows the user to deploy energy-efficient sensor networks and find optimum trajectories for flying around the territory.

The initial data when using static sensors will be the territory image, the maximum coverage radius, the maximum intersection level of the coverage areas, the number of sensors available for use. The result will be a set of pairs of Pareto-optimum values for the coverage radius and the intersection level, which provide energy-efficient coverage of the territory by sensors.

In the case of dynamic sensors, the initial data will be the territory image, the maximum sensor coverage radius, the starting coordinates, and the area of the minimum allowable coverage of the territory by the sensor. As a result, the user will obtain the coordinates of the energy-efficient trajectory and the value of the coverage radius at each node of the trajectory.

The algorithm for finding the parameters of energy-efficient coverage of the territory by static and dynamic sensors can be represented as follows (Fig. 6).

At the first stage, it is necessary to find out the coordinates of the territory border and its area. In turn, the territory can be of any size and shape.

To further build the coverage of the territory, it is necessary to project the territory onto the coordinate plane. To do this, if the territory is depicted on a white background or there is no background, an algorithm for automatically searching for the territory boundaries is proposed. With the specified precision (the default value is 1 pixel), the algorithm, starting from the upper left corner of the uploaded image, goes through all the pixels from left to right. If the color of the next pixel read does not match the color of the previous one, that point is fixed as the left boundary point and written to the LeftPoints array and the traversal stops.

Next, a right-to-left traversal begins to find the right boundary point, which will be written to the RightPoints array. After traversing the entire image, we got two arrays LeftPoints and RightPoints, respectively.

To form an array of border coordinates and save the direction of territory traversal in the resulting array, the first element of the LeftPoints array is assigned to the first element, the next elements will be the elements of the RightPoints array.

After adding all the elements of the RightPoints array, all the elements of the LeftPoints array are added to the resulting array in reverse order. In the resulting array, the first and last elements must match so that the polygon is closed.

Schematically, the proposed algorithm can be depicted as follows (Fig. 7).

The next step is to choose the type of sensors: static or dynamic. Further, in the case of dynamic sensors, it is necessary to set the parameters of the coverage radius, the initial coordinates, and the value of the minimum allowable area covered by the sensor. In the case of static sensors, the user needs to set the coverage radius, the intersection level and the number of available sensors.

After entering the necessary parameters, the user will obtain the result in the form of parameters, which will allow constructing an energy-efficient sensor network while maximizing the coverage of the territory. 


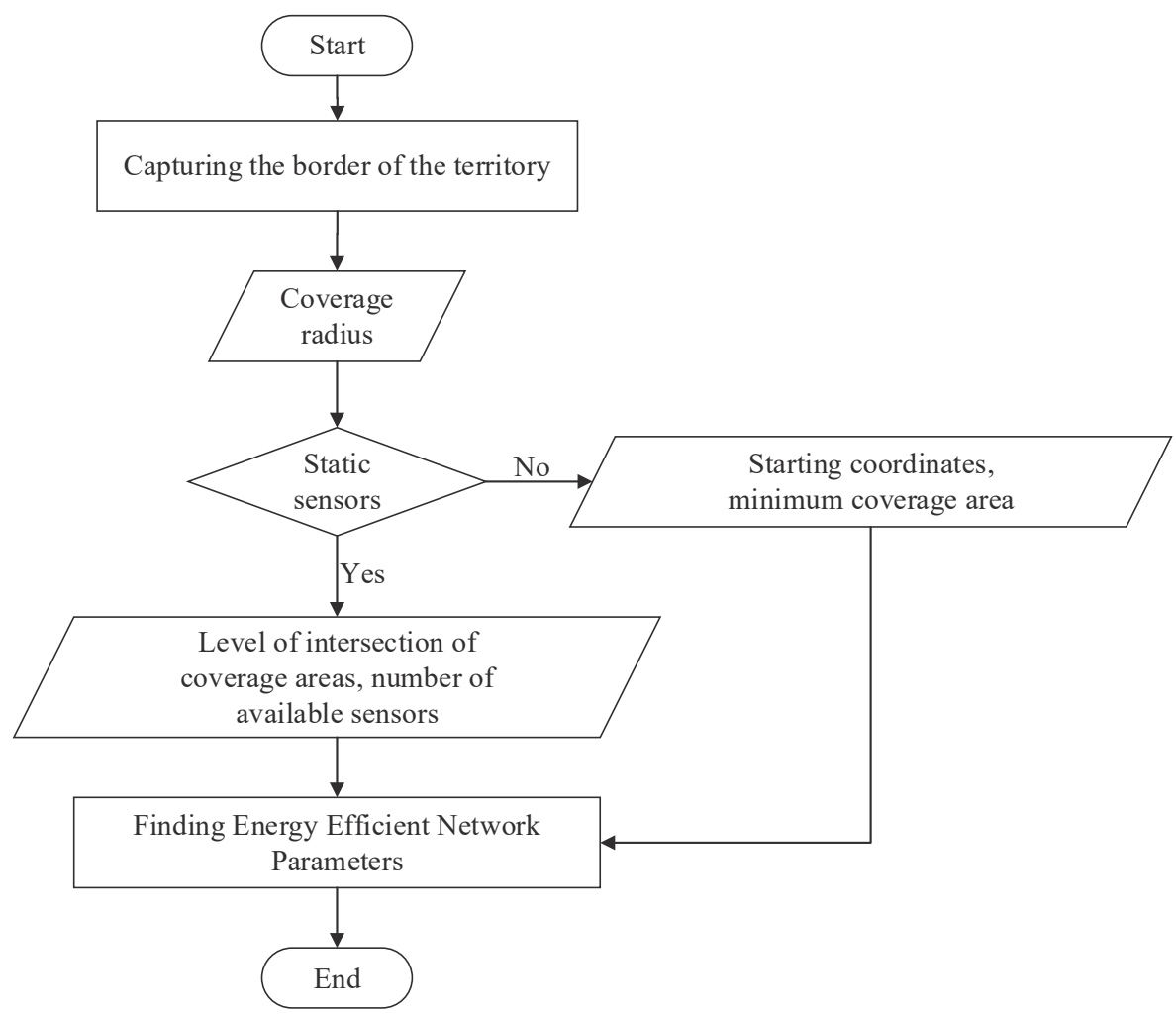

Fig. 6. Block diagram of the algorithm for finding the parameters of an energy-efficient sensor network using static and dynamic sensors

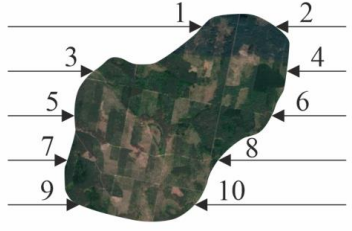

a

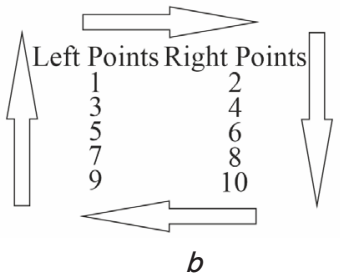

Fig. 7. Example of filling and traversing the arrays of points LeftPoints and RightPoints: $a$ - capturing the area; $b-$ array traversal direction

\section{Discussion of the results}

of the study of finding the parameters of the sensor network based on static and dynamic sensors

To find the optimum value of the territory coverage, the proposed method of automatic capture and calculation of the territory area was used. Initial data: area $-258754 \mathrm{~m}^{2}$, sensors with maximum coverage radius $-r=50 \mathrm{~m}$, number of sensors $-40-50$ pieces. The result of finding the optimum network parameters, which provide the maximum coverage with the minimum power consumption, is shown in Fig. 8.

Analysis of Fig. 8 showed that among the presented pairs of sensor coverage radii and the intersection level of the coverage areas, only seven are suitable if the number of sensors is limited. The initial values of the number of sensors $N_{i n p}$, the power consumption of the original network $E_{i n p}$, the optimum number of sensors $N_{\text {opt }}$, the power consumption of the resulting network $E_{o p t}$, as well as the reduction in power consumption are presented in Table 1.

According to Table 1, limiting the number of network sensors reduced the network power consumption by $18-19 \%$.

In the next experiment, it is necessary to find the optimum trajectory when flying over a given territory. The initial trajectory of the sensor chosen by experts is also known. The sensor coverage radius is $10 \mathrm{~m}$.

Analyzing Fig. 9, we can conclude that the length of the trajectory obtained by the proposed algorithm (Fig. 9, $a$ ) is less than that compiled by the expert (Fig. 9, $b$ ). The reduction in energy consumption using the proposed algorithm is shown in Fig. 10.

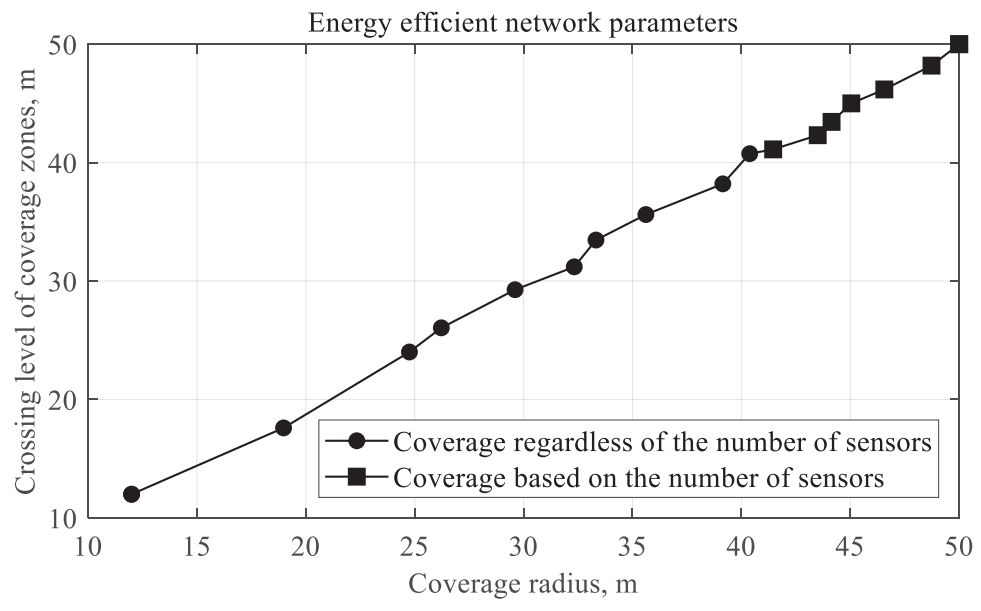

Fig. 8. Sensor network parameters that provide energy-efficient coverage of the territory 
Table 1 ly, the battery life of the sensor increased by 1.8 times, which

Initial and optimum characteristics of the sensor network

\begin{tabular}{|c|c|c|c|c|c|}
\hline$R$ & $N_{\text {inp }}$ & $E_{\text {inp }}$ & $N_{\text {opt }}$ & $E_{\text {opt }}$ & $\begin{array}{c}\text { Reduction in power } \\
\text { consumption }\end{array}$ \\
\hline 50 & 39 & 97,500 & 32 & 80,000 & $18 \%$ \\
\hline 48.3 & 43 & 100314.3 & 35 & 81651.15 & $19 \%$ \\
\hline 46.2 & 47 & 100318.7 & 38 & 81108.72 & $19 \%$ \\
\hline 45 & 49 & 99,225 & 40 & 81,000 & $18 \%$ \\
\hline 44.6 & 50 & 99,458 & 41 & 81555.56 & $18 \%$ \\
\hline 44 & 52 & 100,672 & 42 & 81,312 & $19 \%$ \\
\hline 41 & 60 & 100,860 & 49 & 82,369 & $18 \%$ \\
\hline
\end{tabular}

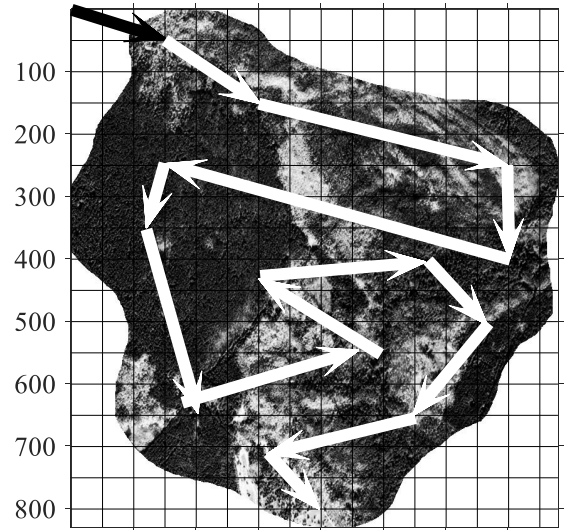

$100 \quad 200 \quad 300 \quad 400 \quad 500 \quad 600 \quad 700$

a

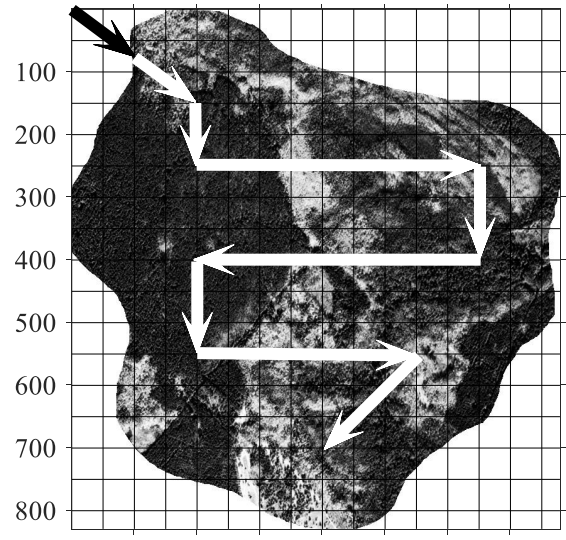

$100 \quad 200 \quad 300 \quad 400 \quad 500 \quad 600 \quad 700$ b
Fig. 9. Trajectories of flying around the territory: $a-$ initial data; $b$ - result of the algorithm

Power consumption when flying around the territory

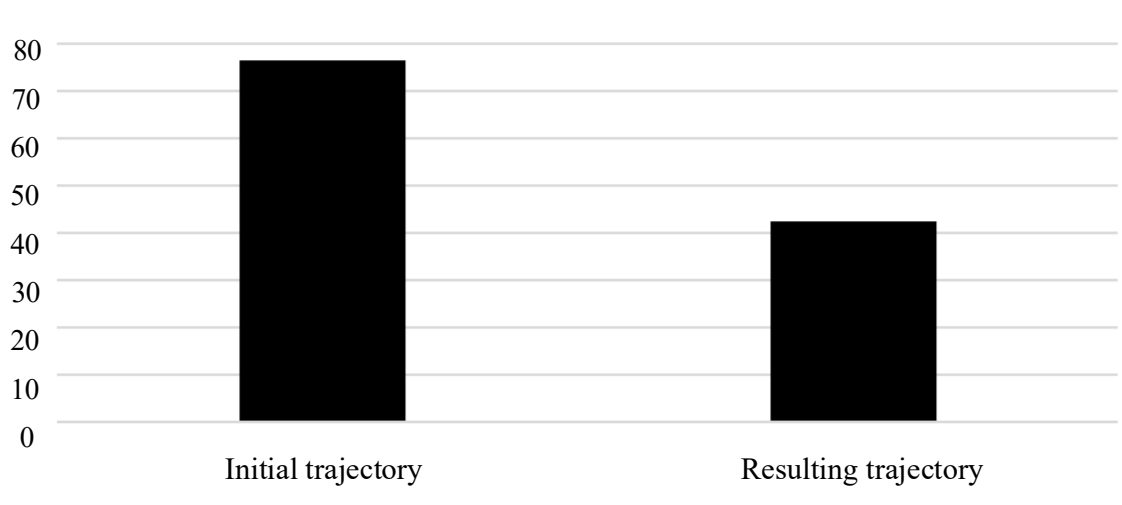

- Power consumption when flying around the territory

Fig. 10. Energy consumption of the sensor for the initial and resulting trajectory
The results show that the conditions for maximizing coverage while minimizing power consumption are satisfied at very close values of the parameters of the coverage radius and the intersection level of the coverage areas (Fig. 8). This is due to the minimization of the uncovered territory. Taking into account the minimization and limitation of the number sensors used, the optimum network parameters are close or equal to the maximum allowable values of the coverage radius and the intersection level of the coverage areas (Fig. 8).

When constructing the trajectory of movement during the flight over the territory, energy consumption was significantly reduced (Fig. 10). In turn, this increased the battery life of the sensor. To apply the results of the study and correctly build the sensor trajectory, the necessary conditions will be:

1. The area of the territory is much larger than the coverage area of the sensor.

2. The boundary of the territory is a closed curve.

Among the nuances, it should be borne in mind that when covering several separate unrelated sections of the territory, the algorithm should be applied for each section separately. It should be noted that the coverage radius and the ability to adjust the coverage radius affect the shape and length of the trajectory, as well as reduce power consumption. The shortcomings include the fact that the direction of the sensor motion changes at right angles only (Fig. 9). As directions for further development of the study, one should consider increasing the number of directions of sensor movement and the possibility of parallel use of several sensors during trajectory construction.

The proposed scheme for the practical implementation of the deployment of sensor networks contains a selection of sensor type (static or dynamic). The method used to construct the coverage depends on the selected sensor type. Further studies should consider the possibility of simultaneous use of static and dynamic sensors in the construction of the territory coverage.
The power consumption value was calculated as the power consumption of the sensor during the flight. The calculations were carried out in conventional units with the assumption that the sensor uses one conventional unit of energy consumption per 10 meters of flight. The energy consumption when flying around the territory along the initial trajectory is 76.51 conventional units, and with the resulting trajectory 42.54 conventional units. The presented graph shows that the proposed approach for finding the optimum trajectory of the sensor provides a 1.8-fold reduction in energy consumption when flying around the territory. According-

\section{Conclusions}

1. A modified method for constructing a sensor network in conditions of maximizing coverage while minimizing power consumption has been developed. A distinctive feature of the modified method, in addition to limiting the number of sensors, is the criterion for minimizing the number of sensors used, which is taken into account together with the criteria for minimizing the uncovered territory and minimizing energy consumption. The solution to this problem is provided 
by the construction of energy-efficient sensor networks of maximum coverage of the territory using a minimum number of sensors and reducing energy consumption by $19 \%$.

2. A method for constructing the motion trajectory of sensors, taking into account the minimization of energy consumption has been developed, which allows minimizing the length of the trajectory with adjustment of the coverage radii of the dynamic sensor at each point of the trajectory. This approach minimizes the energy consumption of the sensor during movement and provides maximum coverage of hard-to-reach areas.
3. An algorithm for deploying sensor networks based on optimum energy consumption has been developed, which makes it possible to form optimum network parameters, as well as automate the process of capturing the territory border from an uploaded image with an accuracy of one pixel. The features of the developed algorithm include the possibility of using both static and dynamic sensors, automated capturing of the territory border, a minimum set of initial parameters, the user's ability to select the necessary network parameters among the results obtained when building the coverage.

\section{References}

1. Grimes, C., Dickey, E., Pishko, M. (Eds.) (2005). Encyclopedia of Sensors: 10-Volume Set. Vols. 1-10. The Pennsylvania State University, University Park. Available at: http://www.aspbs.com/eos.html

2. Blaauw, F. J., Schenk, H. M., Jeronimus, B. F., van der Krieke, L., de Jonge, P., Aiello, M., Emerencia, A. C. (2016). Let's get Physiqual An intuitive and generic method to combine sensor technology with ecological momentary assessments. Journal of Biomedical Informatics, 63, 141-149. doi: https://doi.org/10.1016/j.jbi.2016.08.001

3. Internet of Everything izmenit mir k luchshemu (2012). Available at: https://www.g-news.com.ua/news/10-it/-/13936-internet-ofeverything-izmenit-mir-k-luchshemu.html

4. Warneke, B., Last, M., Liebowitz, B., Pister, K. S. J. (2001). Smart Dust: communicating with a cubic-millimeter computer. Computer, 34 (1), 44-51. doi: https://doi.org/10.1109/2.895117

5. Egorov, L. L., Kologrivov, V. A., Melihov, S. V. (2009). Algoritm rascheta zon pokrytiya bazovyh stantsiy sotovoy svyazi. Doklady TUSURa, 19, 15-21.

6. Wu, H., Liu, Z., Hu, J., Yin, W. (2020). Sensor placement optimization for critical-grid coverage problem of indoor positioning. International Journal of Distributed Sensor Networks, 16 (12), 155014772097992. doi: https://doi.org/10.1177/1550147720979922

7. Danyliuk, S. L. (2016). Adaptive ecological monitoring: conceptual approaches. Modern Information Technologies in the Sphere of Security and Defence, 1 (25), 45-48.

8. Astrakova, S. N., Erzinbc, A. I., Zalyubovskiy, V. V. (2009). Sensor networks and covering of plane by discs. Diskretn. Anal. Issled. Oper., 16 (3), 3-19.

9. Petrivskyi, V., Shevchenko, V., Bychkov, O., Brazhenenko, M. (2020). Information Technology of the Increasing Sensors Term of Use Considering Their Movement. 2020 IEEE XVIth International Conference on the Perspective Technologies and Methods in MEMS Design (MEMSTECH). doi: https://doi.org/10.1109/memstech49584.2020.9109431

10. Petrivskyi, V. Ya., Shevchenko, V. L., Brazhynenko, M. H. (2019). Zbilshennia chasu roboty datchykiv shliakhom rehuliuvannia enerhovytrat. Systemy Obrobky Informatsiyi, 3 (158), 36-41.

11. Luo, C., Chen, W., Li, D., Wang, Y., Du, H., Wu, L., Wu, W. (2021). Optimizing flight trajectory of UAV for efficient data collection in wireless sensor networks. Theoretical Computer Science, 853, 25-42. doi: https://doi.org/10.1016/j.tcs.2020.05.019

12. Haider, S. K., Jiang, A., Almogren, A., Rehman, A. U., Ahmed, A., Khan, W. U., Hamam, H. (2021). Energy Efficient UAV Flight Path Model for Cluster Head Selection in Next-Generation Wireless Sensor Networks. Sensors, 21 (24), 8445. doi: https://doi.org/ $10.3390 / \mathrm{s} 21248445$

13. Amar, M. A., Khaznaji, W., Horchani, L. (2020). PTSP Solution Strategy for Motion Trajectory of UAV in Ubiquitous Sensor Network. Procedia Computer Science, 176, 3191-3199. doi: https://doi.org/10.1016/j.procs.2020.09.130

14. Ghosh, N., Sett, R., Banerjee, I. (2017). An efficient trajectory based routing scheme for delay-sensitive data in wireless sensor network. Computers \& Electrical Engineering, 64, 288-304. doi: https://doi.org/10.1016/j.compeleceng.2017.06.003

15. Miles, J., Kamath, G., Muknahallipatna, S., Stefanovic, M., Kubichek, R. F. (2013). Optimal trajectory determination of a single moving beacon for efficient localization in a mobile ad-hoc network. Ad Hoc Networks, 11 (1), 238-256. doi: https://doi.org/ 10.1016/j.adhoc.2012.05.009

16. Petrivskyi, V. Y., Shevchenko, V. L., Bychkov, O. S., Loza, V. M. (2020). Information technology of territory covering by sensors with the constant intersection level and cost minimization. Collection of Scientific Works of the Military Institute of Kyiv National Taras Shevchenko University, 68, 65-72. doi: https://doi.org/10.17721/2519-481x/2020/68-07

17. Petrivskyi, V., Shevchenko, V., Bychkov, O., Brazhenenko, M., Petrov, P. (2021). CAD technology for optimal territory covering. 2021 IEEE 16th International Conference on the Experience of Designing and Application of CAD Systems (CADSM). doi: https://doi.org/10.1109/cadsm52681.2021.9385223

18. Novikova, N. M., Pospelova, I. I., Zenyukov, A. I. (2017). Method of convolution in multicriteria problems with uncertainty. Journal of Computer and Systems Sciences International, 56 (5), 774-795. doi: https://doi.org/10.1134/s1064230717050082

19. Alperen, Y., Sertac, C. (2020). Multi objective optimization of a micro-channel heat sink through genetic algorithm. International Journal of Heat and Mass Transfer, 146, 118847. doi: https://doi.org/10.1016/j.ijheatmasstransfer.2019.118847

20. Bondarenko, M. F., Bilous, N. V., Rutkas, A. H. (2004). Kompiuterna dyskretna matematyka. Kharkiv: Kompanyia SMYT, 480.

21. Murty, M. R., Thain, N. (2007). Pick's Theorem via Minkowski's Theorem. The American Mathematical Monthly, 114 (8), $732-736$. doi: https://doi.org/10.1080/00029890.2007.11920465 